\title{
STUDY OF THE $\pi^{+} \pi^{-} \gamma$ SYSTEM CENTRALLY PRODUCED
}

IN THE REACTION $p p \rightarrow p_{f}\left(\pi^{+} \pi^{-} \gamma\right) p_{s}$ AT $300 \mathrm{GeV} / \mathrm{c}$

\section{WA76 Collaboration}

Athens-Bari-Birmingham-CERN-Collège de France

T.A. Armstrong ${ }^{4 a}$, R.P. Barnes ${ }^{3}$, M. Benayoun ${ }^{5}$, W. Beusch ${ }^{4}$, I.J. Bloodworth ${ }^{3}$, J.N. Carney ${ }^{3}$, D. Di Bari ${ }^{2}$, C.J. Dodenhoff ${ }^{3}$, C. Evangelista ${ }^{2}$, D. Evans ${ }^{3}$, B. Ghidini ${ }^{2}$, B.R. French ${ }^{4}$, M. Girone ${ }^{2}$, A. Jacholkowski ${ }^{4}$, J.B. Kinson ${ }^{3}$, A. Kirk ${ }^{4}$, K. Knudson ${ }^{4}$, V. Lenti ${ }^{2}$, V. Manzari ${ }^{2}$, F. Navach ${ }^{2}$, A. Palano ${ }^{2}$, E. Quercigh ${ }^{4}$, M. Sené ${ }^{5}$, R. Sené ${ }^{5}$, G. Vassiliadis ${ }^{1}$, O. Villalobos Baillie ${ }^{3}$, M.F. Votruba ${ }^{3}$

\begin{abstract}
The reaction $p p \rightarrow p_{f}\left(\pi^{+} \pi^{-} \gamma\right) p_{s}$, where the $\pi^{+} \pi^{-} \gamma$ system is centrally produced, has been studied at $300 \mathrm{GeV} / \mathrm{c}$. The $\pi^{+} \pi^{-} \gamma$ mass spectrum shows evidence for a $\rho^{0} \gamma$ decay mode of the $\eta^{\prime}(958)$ and $f_{1}(1285)$. The branching ratio $\left(f_{1}(1285) \rightarrow \eta \pi^{+} \pi^{-}\right) /\left(f_{1}(1285) \rightarrow\right.$ $\left.\rho^{0} \gamma\right)$ is found to be $4.2 \pm 1.0$. No evidence is found for a $\rho^{0}(770) \gamma$ decay mode of the $E / f_{1}(1420)$ for which an upper limit B.R. $\left(E / f_{1}(1420) \rightarrow \rho^{0} \gamma\right)<0.08$ at $95 \%$ C.L. is found.
\end{abstract}

Submitted to Zeitschrift für Physik C

1) Athens University, Nuclear Physics Department, Athens, Greece

2) Dipartimento di Fisica dell'Università and Sezione INFN, Bari, Italy

3) University of Birmingham, Physics Department, Birmingham, U.K.

4) CERN, European Organization for Nuclear Research, Geneva, Switzerland

5) Collège de France, Paris, France

a) Present address: Pennsylvania State University, University Park, USA 


\section{INTRODUCTION}

The radiative decays of mesons have an important role to play in particle physics since they give information on the quark content of the mesons being studied. Therefore the study of the radiative decays of meson resonances is particularly useful in the search for glueball and hybrid states.

The radiative decay of the gluonium candidate $\iota / \eta(1440) \rightarrow \rho^{0} \gamma$ has been searched for in the process $J / \psi \rightarrow \gamma \rho^{0} \gamma, \rho^{0} \rightarrow \pi^{+} \pi^{-}$in several experiments [1]. They all observe a peak in the $\rho^{0} \gamma$ mass spectrum, but too low in mass and too wide to be identified with the $\iota / \eta(1440)$, discovered in the radiative $J / \psi$ decay to $\mathrm{K} \overline{\mathrm{K}} \pi$. However, a recent Mark III study of radiative $J / \psi$ decay to $\rho^{0} \gamma$ [2] finds two peaks in the $\rho^{0} \gamma$ mass distribution: the lower mass state has parameters consistent with those of the $f_{1}(1285)$, while for the higher mass peak the hypotheses of being the axial meson $E / f_{1}(1420)$ or the pseudoscalar state $\iota / \eta(1440)$ are both possible.

The nature of the $\mathrm{E} / f_{1}(1420)$ meson has remained problematical, despite many at-

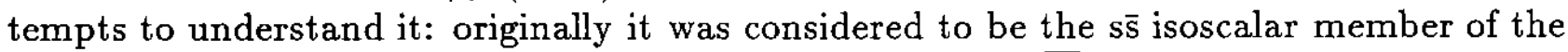
ground state $1^{++}$nonet, as suggested from its dominant $K^{*} \bar{K}$ decay mode, but recently it. has been proposed as a candidate hybrid [3] or a four quark state $[4,5]$. Its interpretation as a $\mathrm{q} \overline{\mathrm{q}}$ state presents many difficulties: among these is the fact that the $E / f_{1}(1420)$ is not

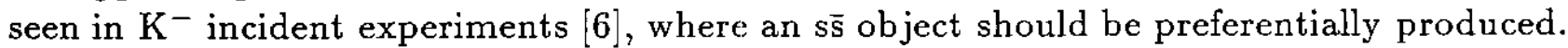
Instead a different $1^{++}$state, the $f_{1}(1530)$ has been observed. The $E / f_{1}(1420)$ is produced

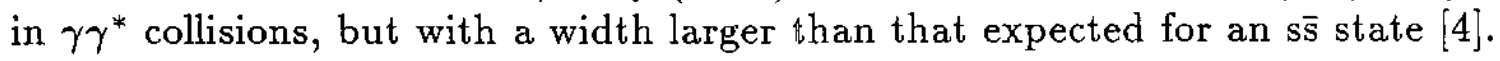

The $E / f_{1}(1420)$ has been observed decaying to $K \bar{K} \pi$, with good statistics, in central production by experiment WA76 [7]. It has also been searched for, in the same experiment, in the $2 \pi^{+} 2 \pi^{-}$[8] and $\eta \pi^{+} \pi^{-}$[9] decay modes with negative results. It is therefore of interest to investigate the $\rho^{0} \gamma$ final state in the same experiment in order to add information regarding the nature of the states observed in this mass region.

This paper studies the centrally produced exclusive final states formed in the reaction:

$$
p p \rightarrow p_{f}\left(\pi^{+} \pi^{-} \gamma\right) p_{s}
$$

at $300 \mathrm{GeV} / \mathrm{c}$, where the subscripts $f$ and $s$ indicate the fastest and the slowest particles in the laboratory respectively. The data come from experiment WA76 which has been performed using the CERN Omega Spectrometer. Details on the layout of the apparatus, trigger conditions and data processing have been given in a previous publication [10].

\section{SELECTION OF THE REACTION $p p \rightarrow p_{f}\left(\pi^{+} \pi^{-} \gamma\right) p_{s}$}

Candidates for reaction (1) have been selected from the sample of events having four outgoing charged tracks and only one $\gamma$ having an energy greater than $1.5 \mathrm{GeV}$ detected in the electromagnetic calorimeters [11], by imposing the following cuts on the components of 
missing momentum: $\mid$ missing $P_{x}|<16.0 \mathrm{GeV} / \mathrm{c}$,$| missing P_{y}|<0.12 \mathrm{GeV} / \mathrm{c}$,$| missing P_{z} \mid<$ $0.06 \mathrm{GeV} / \mathrm{c}$. Showers associated with charged track impacts on the $\gamma$ calorimeters were removed. The energy balance was required by demanding the Ehrlich mass squared [12] to be in the range from -0.14 to $0.16 \mathrm{GeV}^{2}$. The charged particles were then required to have a mass consistent with being a pion as given by the Cerenkov information. Events where the slow particle was identified as a $\pi^{+}$were antiselected using pulse height momentum correlation. Only $\gamma$ 's with a centre of mass rapidity less than 1.7 were retained in order to remove $\gamma$ 's from diffractive processes. A similar cut was also used to antiselect diffractive $\pi^{+'}$ s.

Possible sources of contamination to reaction (1) include the $\pi^{+} \pi^{-}$channel with a background $\gamma$ in the beam region and the reaction:

$$
p p \rightarrow p_{f}\left(\pi^{+} \pi^{-} \pi^{0}\right) p_{s}
$$

in which one $\gamma$ from the $\pi^{0}$ decay has escaped detection. To remove background $\gamma$ 's the energy of the $\gamma$ was required to be greater than $2.5 \mathrm{GeV}$ if it was in a circle of radius $30 \mathrm{~cm}$ in the middle of the plane of the electromagnetic calorimeter. Events from the exclusive reaction $p p \rightarrow p_{f}\left(\pi^{+} \pi^{-}\right) p_{s}$ have been also removed.

The $\pi^{+} \pi^{-} \gamma$ mass spectrum is shown in fig. 1(a) where several structures can be seen. The two lowest mass peaks are due to the reflections of $\eta$ and $\omega(783)$ coming from the $\pi^{+} \pi^{-} \pi^{0}$ channel where one $\gamma$ is missing. There are also two enhancements, one at 0.96 $\mathrm{GeV}$ due to the $\eta^{\prime}(958)$ and one at $1.27 \mathrm{GeV}$ which could be due to the $f_{1}(1285)$.

Fig. 1(b) shows the $\pi^{+} \pi^{-}$mass spectrum from reaction (1) where a $\rho^{0}(770)$ signal can be seen. Requiring $0.62 \leq m\left(\pi^{+} \pi^{-}\right) \leq 0.92 \mathrm{GeV}$ gives the $\rho^{0}(770) \gamma$ effective mass distribution shown in fig. 1 (c). The two signals at 0.96 and $1.27 \mathrm{GeV}$ can clearly be seen.

In order to reduce the background from the $\pi^{+} \pi^{-} \pi^{0}$ channel we have adopted the following procedure. A fit was performed to events which were candidates to reaction (1) assuming that they were all coming from reaction (2). The missing $\gamma$ was reconstructed by assigning to it the missing transverse momentum and computing the longitudinal momentum using the constraint that it was coming from a $\pi^{0}$ decay. The events which did not have a physical solution to this assumption (13\%) were considered to belong to reaction (1). For these events, the $\rho^{0} \gamma$ mass spectrum is shown in fig. 2(a). For the remaining events we studied the $\cos \phi$ distribution, $\phi$ being the angle between the measured $\gamma$ and the direction of flight of the $\pi^{0}$, in the $\pi^{0}$ rest frame. This distribution is expected to be flat, due to the pseudoscalar nature of the decaying particle. An accumulation of events was observed at large values of $\cos \phi$, which we interpreted as due to the presence of the $\pi^{+} \pi^{-} \gamma$ channel. If we select events having $\cos \phi>0.7$ we obtain the $\rho^{0} \gamma$ mass distribution shown in fig. 2(b). For the remaining events we show in fig 2(c) the corresponding $\rho^{0} \gamma$ mass spectrum, where there is little evidence for an $\eta^{\prime}(958)$ signal. We have, therefore, antiselected these events ( $12 \%$ of the total). After these selections we obtained a final sample of 8383 events which are candidates for reaction (1) for which the $\rho^{0}(770) \gamma$ mass spectrum is shown in fig. 3. The shaded distribution in fig. 3 shows the $\rho^{0}(770) \gamma$ mass spectrum from the $\pi^{+} \pi^{-} \pi^{0}$ channel, obtained by using events from reaction (2) after having removed the 
lowest energy $\gamma$. This distribution shows no structure in the $1.27 \mathrm{GeV}$ region, therefore the peak observed in the $\rho^{0}(770) \gamma$ mass distribution cannot be due to a reflection from the $\pi^{+} \pi^{-} \pi^{0}$ final state.

\section{STUDY OF THE t DEPENDENCE}

A study of the $t$ dependence of the signals has been performed by dividing the data sample into two $t$ regions. Here, $t=\left|t_{1}+t_{2}\right|$ where $t_{1}$ and $t_{2}$ are the four momentum transfers from the upper and the lower proton vertices respectively. Fig. 4(a,b) shows the $\rho^{0}(770) \gamma$ effective mass distributions for $t<0.3 \mathrm{GeV}^{2}$ and for $t>0.3 \mathrm{GeV}^{2}$. We observe an increase of the signal/background ratio for both $\eta^{\prime}(958)$ and $f_{1}(1285)$ for $t>0.3 \mathrm{GeV}^{2}$.

A fit was performed to the high $t$ mass spectrum in order to determine the parameters of the higher mass enhancement. The $f_{1}(1285)$ has been fitted with a Breit-Wigner convoluted to a Gaussian, where the width of the Breit Wigner has been fixed to the PDG value [13] and the $\sigma$ of the Gaussian which represents the experimental resolution is 25 $\mathrm{MeV}$. The fit gives

$$
m_{f_{1}(1285)}=1270 \pm 6(\text { stat. }) \pm 10(\text { syst. }) \quad \mathrm{MeV}
$$

The systematic error on the mass is due to the fact that there is no kinematical constraint on the energy of the $\gamma$ which is related to the absolute energy calibration of the electromagnetic calorimeters.

In order to measure the $t$ dependence of the observed resonances, we have divided the data into four slices of t. Fig. 5(a-d) shows the $\rho^{0}(770) \gamma$ mass distribution for each $\mathrm{t}$ interval. We observe a different behaviour for $\eta^{\prime}(958)$ and $f_{1}(1285)$ as a function of $t$. The $\mathrm{t}$ distributions for $\eta^{\prime}(958)$ and $f_{1}(1285)$ uncorrected and corrected for geornetrical acceptance are shown in fig. 6(a,b). We observe that the $f_{1}(1285)$ distribution can be described with a simple exponential $\exp (-b t)$ with $b=6.8 \pm 0.7 \mathrm{GeV}^{-2}$, consistent with the results obtained from the study of $f_{1}(1285) \rightarrow \eta \pi^{+} \pi^{-}\left(b=6.5 \pm 0.4 \mathrm{GeV}^{-2}\right)$ and $f_{1}(1285) \rightarrow K_{S}^{0} K^{ \pm} \pi^{\mp}\left(b=7.3 \pm 0.7 \mathrm{GeV}^{-2}\right)[9]$.

Fig. $7(\mathrm{a})$ shows the $\pi^{+} \pi^{-}$vs. $\pi^{+} \pi^{-} \gamma$ scatter plot for events having $t>0.3 \mathrm{GeV}^{2}$ and for a mass of the $\pi^{+} \pi^{-} \gamma$ system above $0.8 \mathrm{GeV}$. Accumulations of events can be seen in the $\rho^{0}(770)$ region of the $\pi^{+} \pi^{-}$effective mass corresponding to the peaks at 0.96 and $1.27 \mathrm{GeV}$. The $\pi^{+} \pi^{-}$effective mass distributions in the $\eta^{\prime}(958)$ region $\left(0.92 \leq m\left(\pi^{+} \pi^{-} \gamma\right) \leq 1.02\right.$ $\mathrm{GeV})$ and the $f_{1}(1285)$ region $\left(1.22 \leq m\left(\pi^{+} \pi^{-} \gamma\right) \leq 1.32 \mathrm{GeV}\right)$ are shown in figs. $7(\mathrm{~b})$ and $7(\mathrm{c})$ respectively. A clear $\rho^{0}(770)$ can be seen in both spectra, showing that the two enhancements at 0.96 and $1.27 \mathrm{GeV}$ have an important $\rho^{0}(770) \gamma$ decay mode. 


\section{STUDY OF THE ANGULAR DISTRIBUTIONS}

An angular analysis of the $\rho^{0}(770) \gamma$ system has been performed by measuring the angle $\theta$ between the $\pi^{+}$and the $\gamma$ in the $\rho^{0}(770)$ rest frame. Fig. 8(a,b) shows the $\cos \theta$ distributions for the $\eta^{\prime}(958)$ and $f_{1}(1285)$ regions. The background was removed by subtracting the corresponding distributions in the two adjacent mass intervals on either side. The distributions were corrected for the geometrical acceptance of the apparatus. For a pseudoscalar resonance decaying to $\rho^{0} \gamma$ the angular distribution should be proportional to $\sin ^{2} \theta$ [14] and, in fact, we obtain a good description of the $\eta^{\prime}(958)\left(\chi^{2} / \mathrm{NDF}=8 / 10\right)$ using this parametrization. The angular distribution of the higher mass state is consistent with being flat and was fitted according to

$$
W(\theta) \propto B_{0} \sin ^{2} \theta+B_{1} \cos ^{2} \theta
$$

where $B_{0}$ and $B_{1}=B_{-1}$ are the helicity 0 and 1 amplitudes describing the decay reaction $X \rightarrow \rho^{0} \gamma$. We obtain a ratio $B_{0} / B_{1}=0.87 \pm 0.50$ with $\chi^{2} / \mathrm{NDF}=10 / 9$.

We conclude that the resonance around $0.96 \mathrm{GeV}$ is compatible with having $J^{P C}=$ $0^{-+}$and a mass consistent with being the $\eta^{\prime}(958)$. The higher mass resonance has a mass consistent within the errors with the $f_{1}(1285)$. The spin analysis indicates that it could be due to an axial meson $\left(f_{1}(1285)\right)$ or to a mixture of pseudoscalar and axial mesons. However, the latter possibility is unlikely since no evidence for pseudoscalar resonances (i.e. $\eta(1270)$ ) has been observed in the study of the $\eta \pi^{+} \pi^{-}$system in the same experiment $[9]$.

\section{MEASUREMENT OF THE BRANCHING RATIOS}

In the same experiment the $\eta^{\prime}(958)$ and the $f_{1}(1285)$ have been observed decaying to $\eta \pi^{+} \pi^{-}$. After having corrected for the geometrical acceptance in the $\eta \pi^{+} \pi^{-}$and $\rho^{0}(770) \gamma$ systems we have computed the branching ratio of the $f_{1}(1285)$ to $\eta \pi^{+} \pi^{-}$and $\rho^{0}(770) \gamma$, using the known branching ratio of the $\eta^{\prime}(958)$ to these two channels [13] as a reference. We obtain

$$
\left(f_{1}(1285) \rightarrow \eta \pi^{+} \pi^{-}\right) /\left(f_{1}(1285) \rightarrow \rho^{0} \gamma\right)=4.2 \pm 1.0
$$

corresponding to $\Gamma_{f_{1}(1285) \rightarrow \rho^{0} \gamma}=2.0 \pm 0.5 \mathrm{MeV}$ in good agreement with the Mark III measurement of $1.7 \pm 0.7$ [2] but somewhat larger than theoretical calculations $[14,15]$.

No evidence is found for a $\rho^{0} \gamma$ decay mode of $\iota / \eta(1440)$ or $E / f_{1}(1420)$. Using the data from $E / f_{1}(1420) \rightarrow K_{s}^{0} K^{ \pm} \pi^{\mp}$ in this experiment [7] we measure an upper limit B.R. $\left(E / f_{1}(1420) \rightarrow \rho^{0} \gamma\right)<0.08$ at $95 \%$ C.L. 


\section{CONCLUSIONS}

In conclusion, the analysis of the centrally produced $\pi^{+} \pi^{-} \gamma$ channel shows evidence for a $\rho^{0} \gamma$ decay mode of the $\eta^{\prime}(958)$ and $f_{1}(1285)$. The branching ratio $\left(f_{1}(1285) \rightarrow\right.$ $\left.\eta \pi^{+} \pi^{-}\right) /\left(f_{1}(1285) \rightarrow \rho^{0} \gamma\right)$ is found to be $4.2 \pm 1.0$. No evidence is found for a $\rho^{0}(770) \gamma$ decay mode of the $E / f_{1}(1420)$ for which an upper limit $B . R .\left(E / f_{1}(1420) \rightarrow \rho^{0} \gamma\right)<0.08$ at $95 \%$ C.L. is found.

\section{ACKNOWLEDGMENTS}

We acknowledge T.H. Burnett and W. Toki for useful suggestions. 


\section{REFERENCES}

[1] C. Edwards, Ph.D Thesis, CALT-68-1165 (1985).

J.D. Richman, Ph.D Thesis, CALT-68 1231 (1985).

J. Augustin et al., Phys. Rev. D42 (1990) 10.

[2] D. Coffman et al., Phys. Rev. D41 (1990) 1410.

[3] S. Ishida et al., Progress of Theoretical Phys. 82 (1989) 119.

[4] D.O. Caldwell, Mod. Phys. Lett. A2 (1987) 771;

[5] R.S. Longacre, Phys, Rev. D42 (1990) 874.

[6] Ph. Gavillet et al., Z. Phys. C16 (1982) 119;

D. Aston et al., Phys. Lett. 201B (1988) 573.

[7] T. Armstrong et al., Phys. Lett. 146B (1984) 273.

T. Armstrong et al., Z. Phys. C34 (1987) 23.

T. Armstrong et al., Phys. Lett. 221B (1989) 221.

[8] T. Armstrong et al., Phys. Lett. 228B (1989) 536.

[9] T. Armstrong et al., CERN/PPE 91-93, 4 June 1991. Submitted to Z. Phys. C.

[10] T. Armstrong et al., Nucl. Instr. and Meth. A276 (1989) 165.

[11] H. Burmeister et al., Nucl. Instr. and Meth. A225 (1984) 530;

M. Bonesini et al., Nucl. Instr. and Meth. A261 (1987) 471.

[12] R. Ehrlich et al., Phys. Rev. Lett. 20 (1968) 686.

[13] Review of Particle Properties. Phys. Lett. B239 (1990)

[14] J. Babcock and J.L. Rosner Phys. Rev. D14 (1976) 1286.

[15] M.K. Volkov, Sov. J. Nucl. Phys. 40 (1984) 622;

S. Ishida et al., Phys. Rev. D40 (1989) 1497. 


\section{Figure captions}

Fig. 1 (a) $\pi^{+} \pi^{-} \gamma$ effective mass distribution;

(b) $\pi^{+} \pi^{-}$effective mass distribution;

(c) $\rho^{0}(770) \gamma$ effective mass distribution.

Fig. 2 (a) $\rho^{0}(770) \gamma$ effective mass distribution. for events not fitting the $\pi^{+} \pi^{-} \pi^{0}$ hypothesis (see text);

(b) $\rho^{0}(770) \gamma$ effective mass distribution for events having $\cos \phi>0.7$;

(c) $\rho^{0}(770) \gamma$ effective mass distribution for $\cos \phi \leq 0.7$.

Fig. $3 \rho^{0}(770) \gamma$ effective mass distribution. The shaded histogram represents the $\rho^{0}(770) \gamma$ background from $\pi^{+} \pi^{-} \pi^{0}$ with arbitrary normalization.

Fig. $4 \rho^{0}(770) \gamma$ effective mass for (a) $t<0.3 \mathrm{GeV}^{2}$ and (b) $t>0.3 \mathrm{GeV}^{2}$.

Fig. $5 \rho^{0} \gamma$ effective mass distributions in different $t$ intervals. (a) $0.1<t<0.2$, (b) $0.2<$ $t<0.3$, (c) $0.3<t<0.5$ and (d) $0.5<t<1.0 \mathrm{GeV}^{2}$.

Fig. $61 / \mathrm{t} \mathrm{dN} / \mathrm{dt}$ distributions (in arbitrary units) for: (a) $\eta^{\prime} \rightarrow \rho^{0} \gamma$, (b) $f_{1}(1285) \rightarrow \rho^{0} \gamma$. Open points: uncorrected data; black points: data corrected by geometrical acceptance.

Fig. 7 (a) $\pi^{+} \pi^{-}$vs. $\pi^{+} \pi^{-} \gamma$ scatter plot;

(b) $\pi^{+} \pi^{-}$effective mass distribution in the $\eta^{\prime}(958)$ region;

(c) $\pi^{+} \pi^{-}$effective mass distribution in the $f_{1}(1285)$ region.

The distributions are for $t>0.3 \mathrm{GeV}^{2}$.

Fig. 8 Fit of the $\rho^{0}(770) \gamma$ angular distributions (see text) for: (a) $\eta^{\prime}(958)$; (b) $f_{1}(1285)$. 

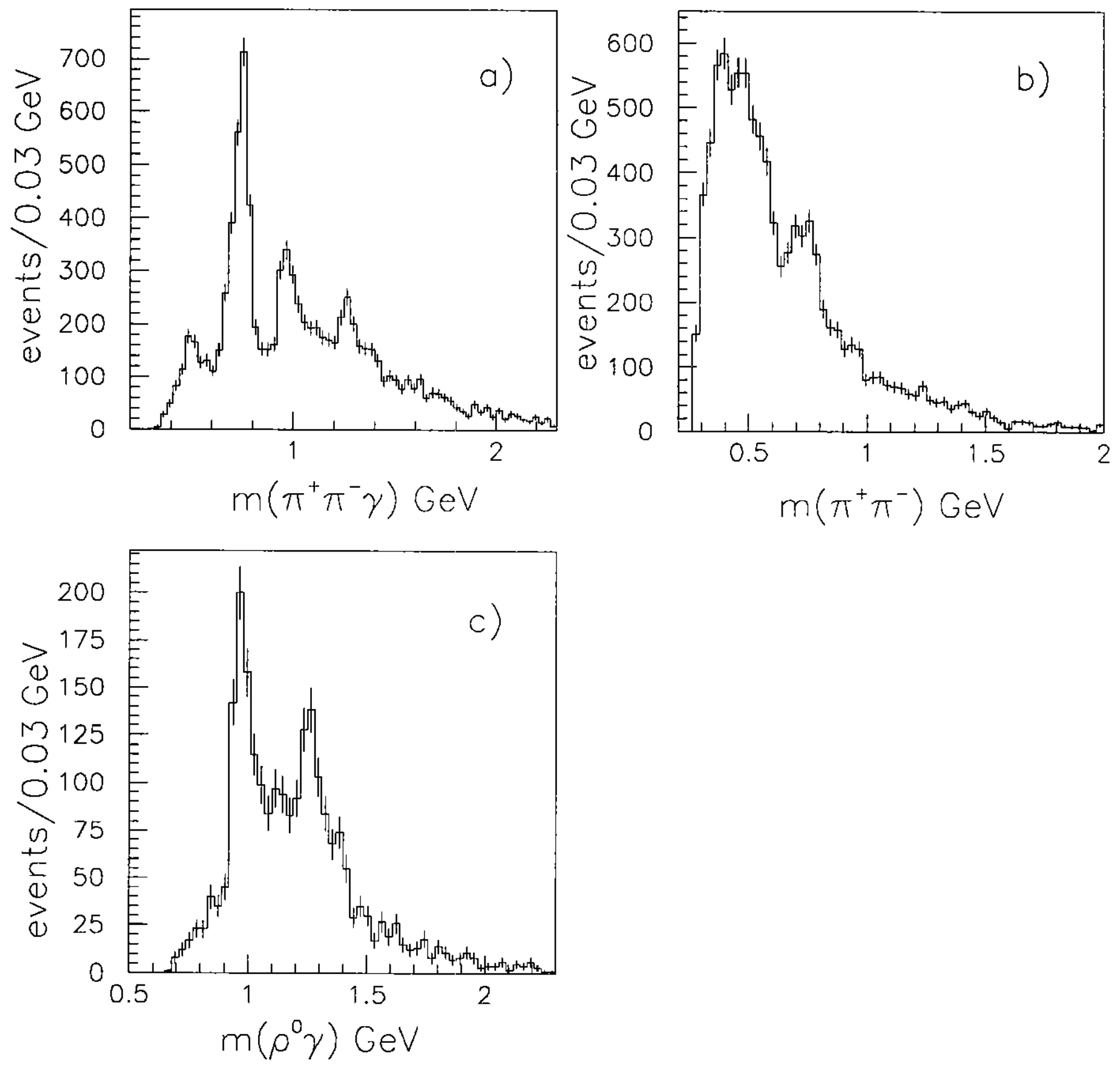

Fig. 1 

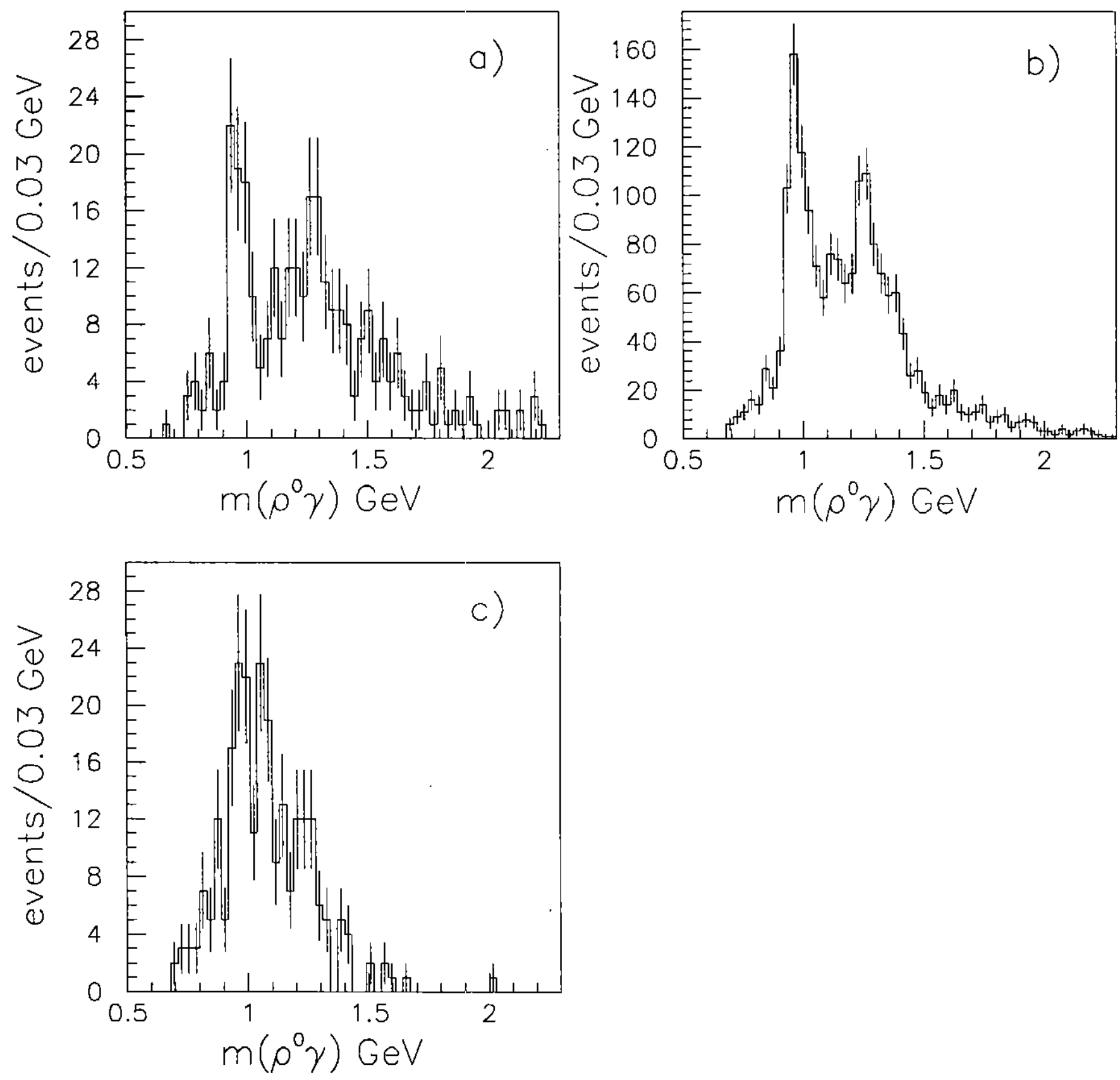

Fig. 2 


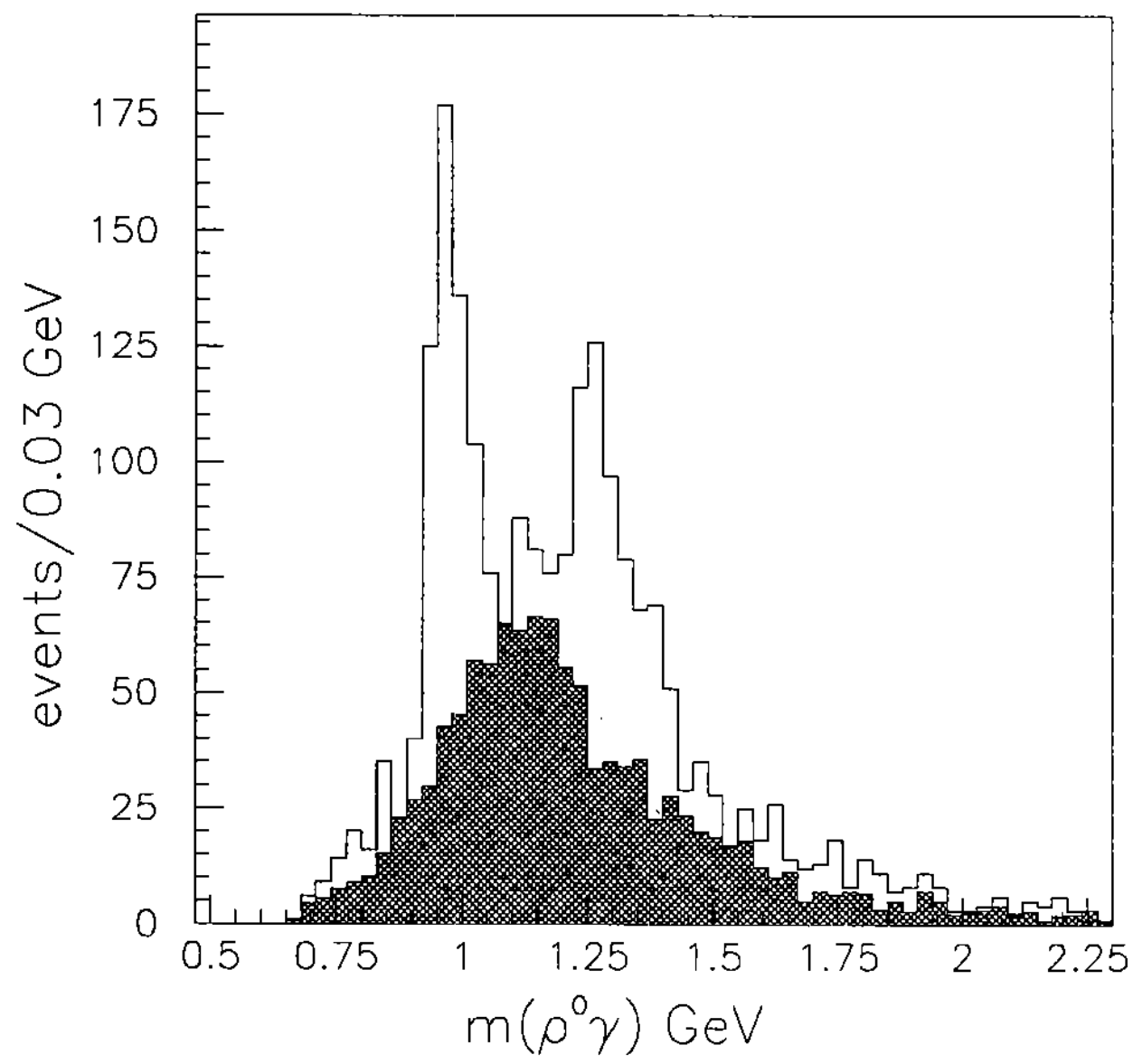

Fig. 3 

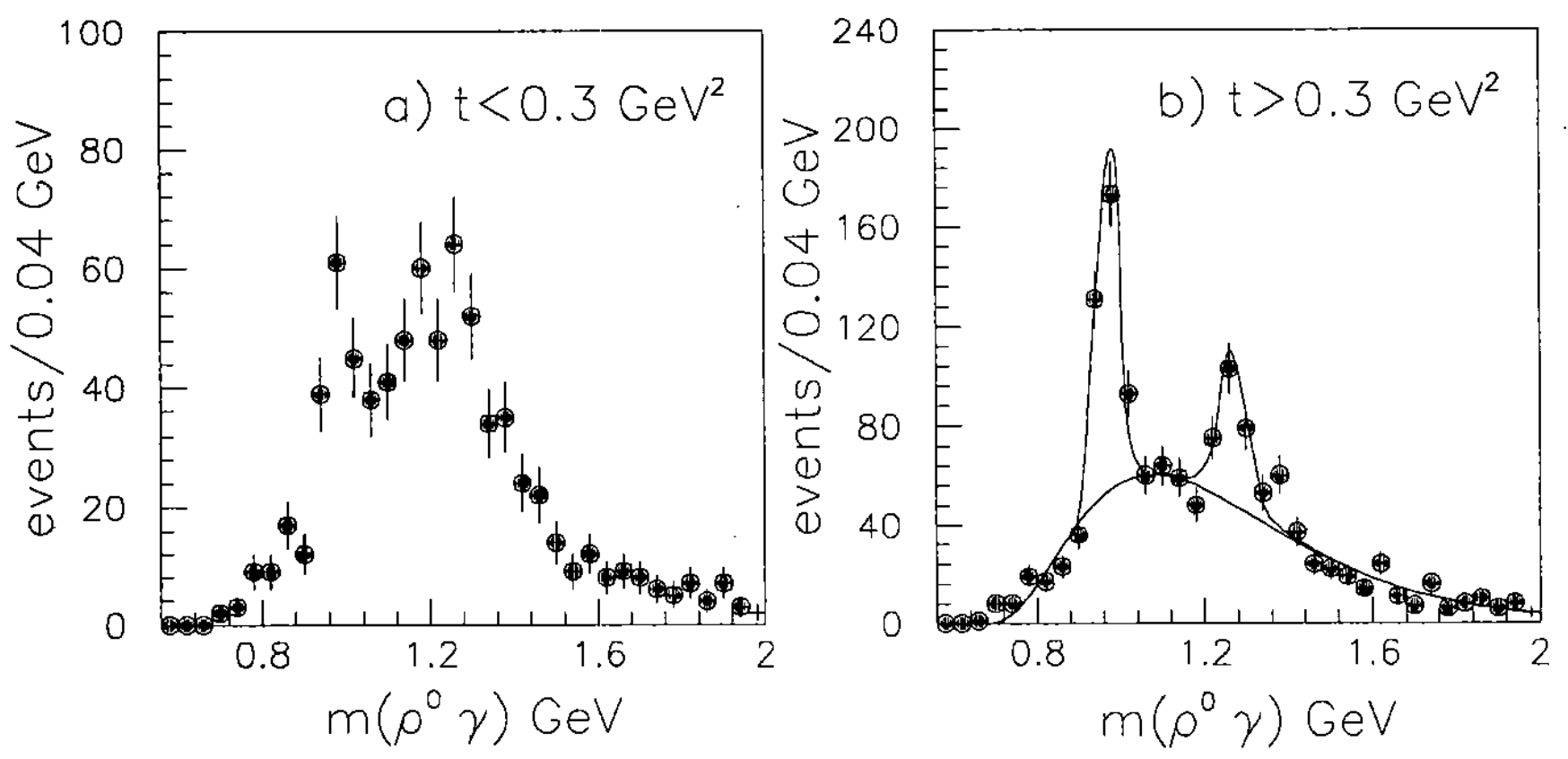

Fig. 4 

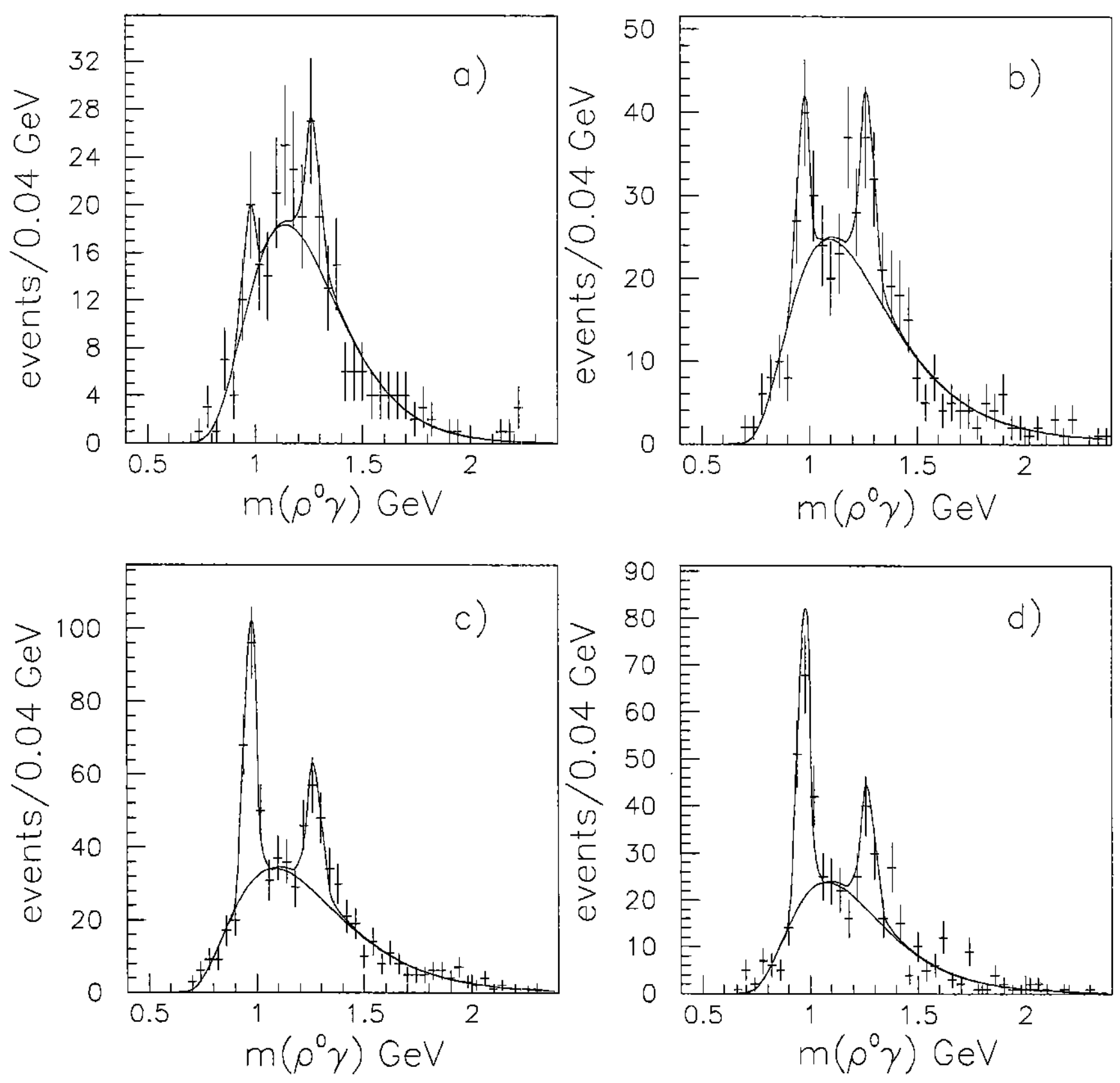

Fig. 5 

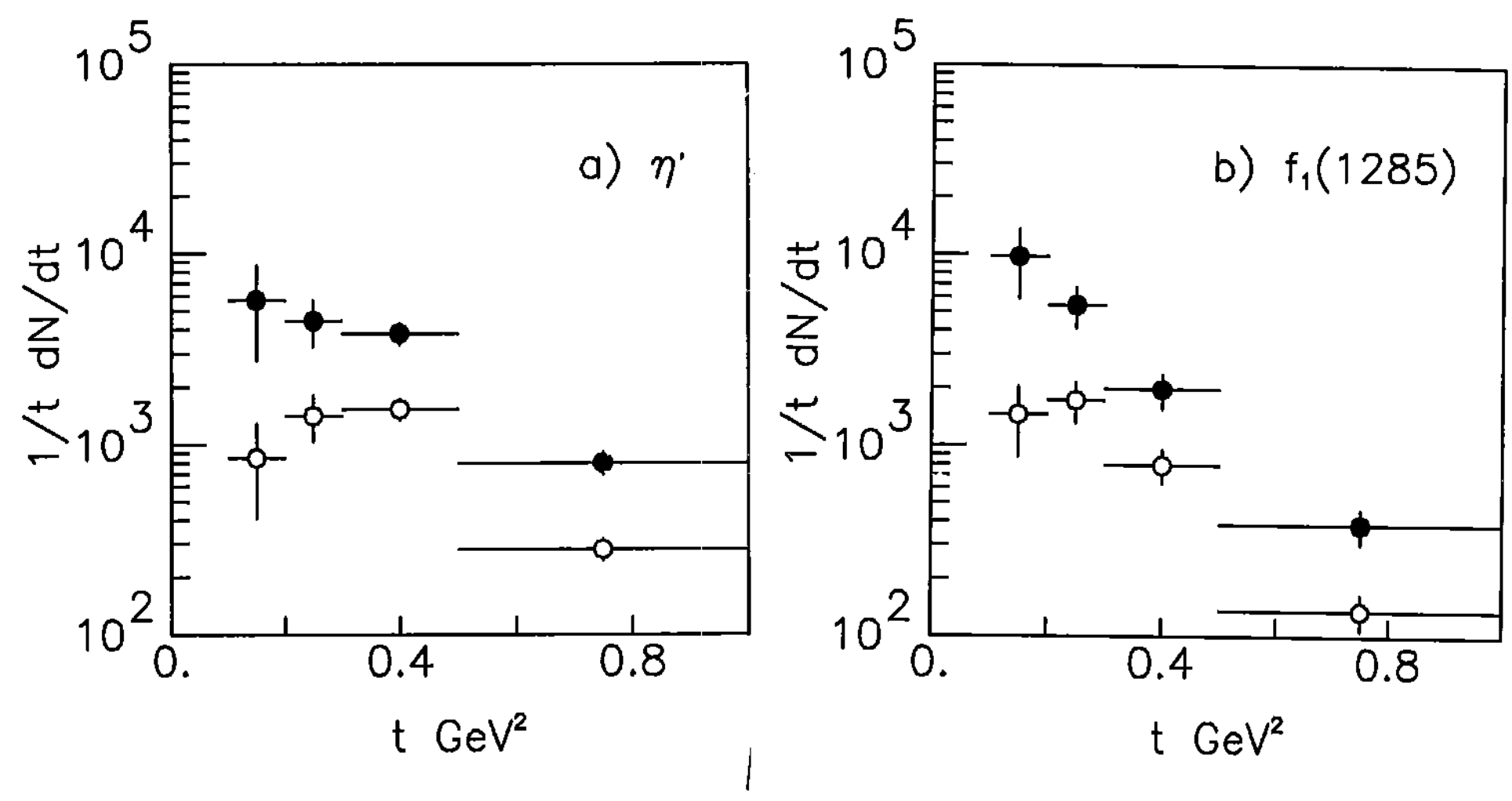

Fig. 6 

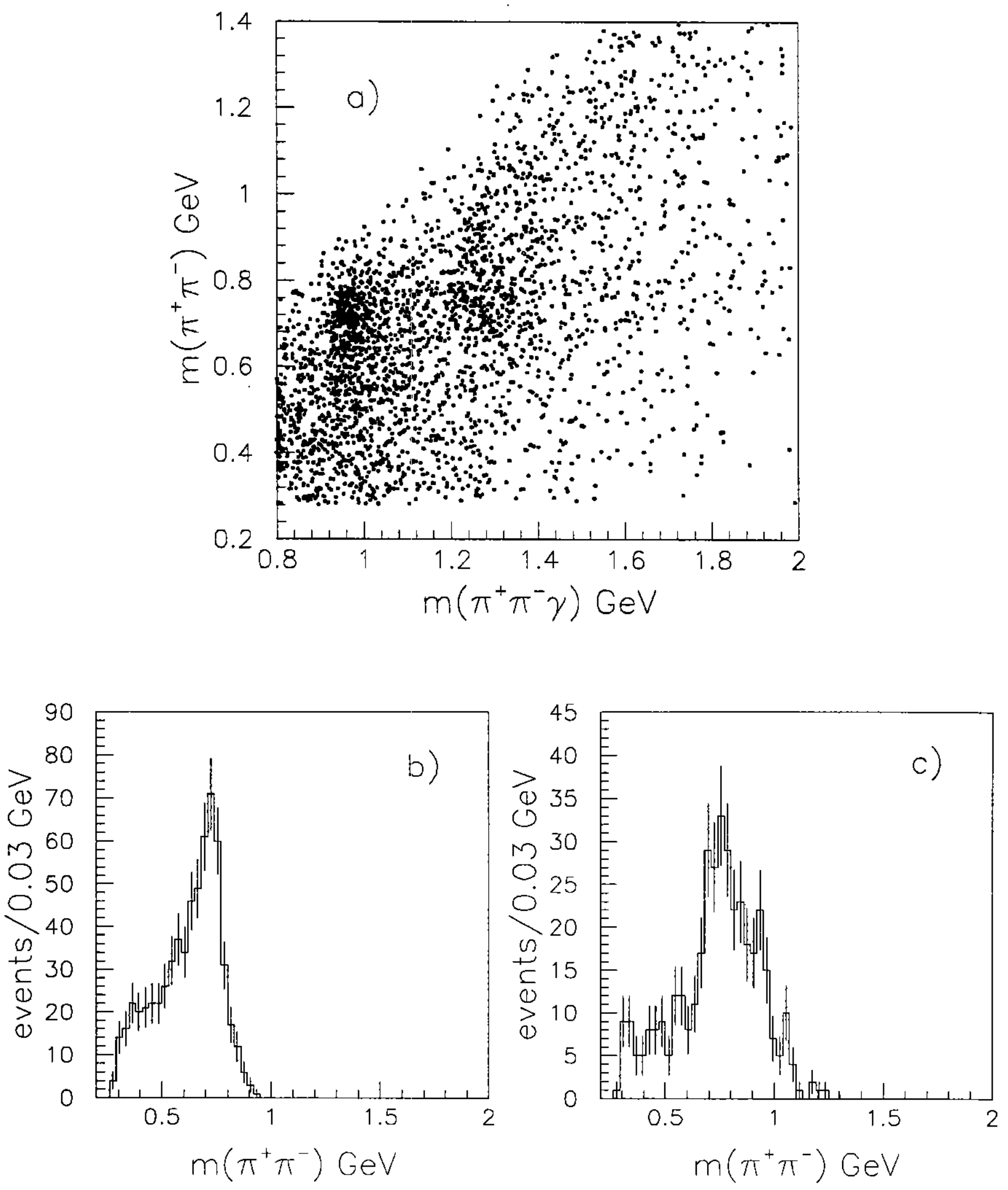

Fig. 7 

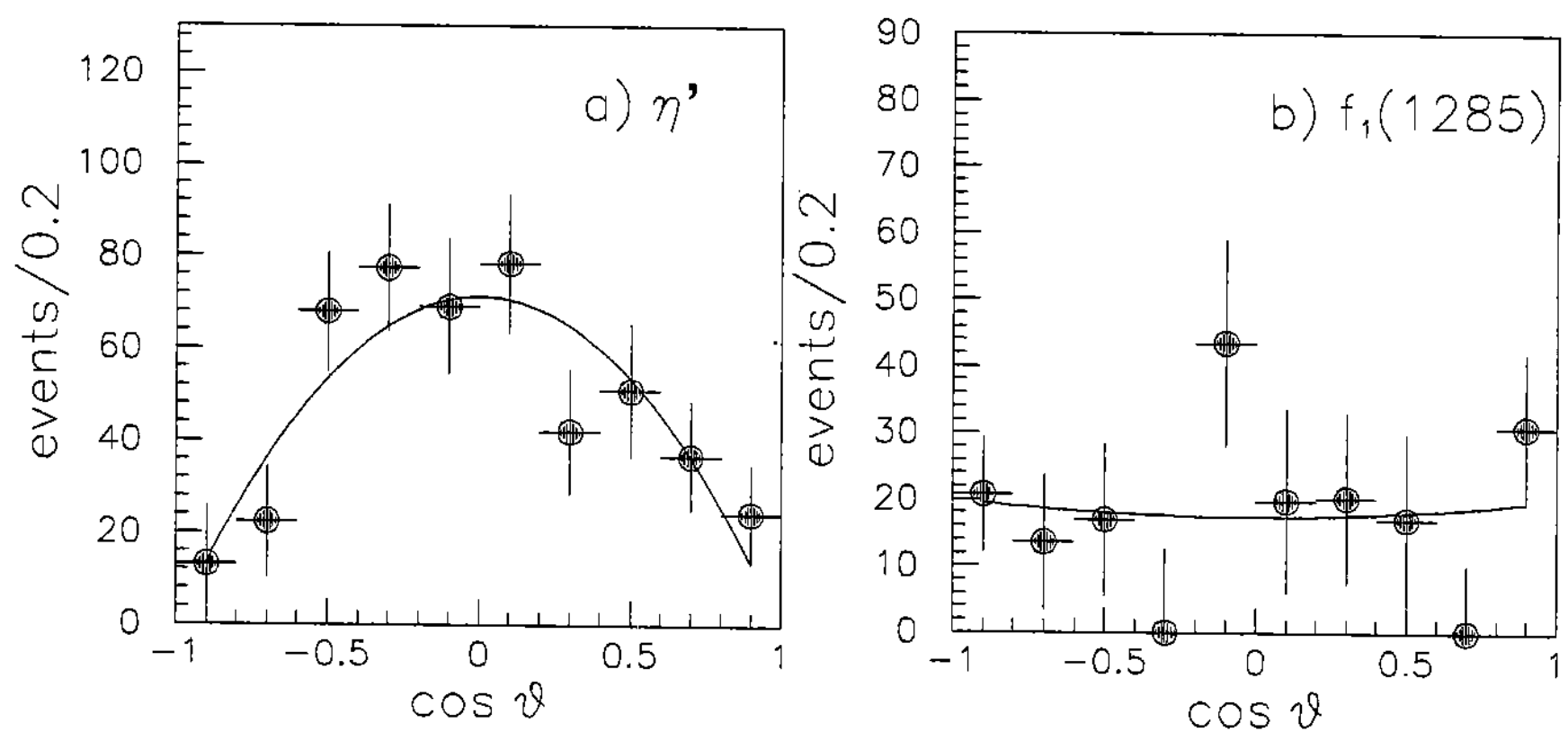

Fig. 8 